\title{
Probing the Optical Pressure of Pathogen-Exposed Macrophages on a Microfluidic Chip
}

\author{
Greg E. Collins ${ }^{1}$, Dhanya Haridas ${ }^{2}$, Daniel E. Barlow ${ }^{1}$, Braden C. Giordano ${ }^{1}$, Qin Lu ${ }^{1}$ \\ 1 Naval Research Laboratory, 4555 Overlook Ave., S.W., Washington D.C. 20375 USA, \\ 2 American Society for Engineering Education, 1818 N Street, N.W. Washington DC 20036 USA \\ qin.lu@nrl.navy.mil
}

\begin{abstract}
:
Optical force can be utilized to achieve label-free sensing and separation by utilizing this force in conjunction with the drag forces from a fluid inside the channel of a microfluidic chip. The custom microfluidic chip discussed here is comprised of five layers, incorporating a 3D hydrodynamic focusing nozzle, an injection channel, and an exit channel which can be branched into two or more channels for cell sorting and collection. The exposure of macrophages, a type of innate immune cell which plays an important role in the body's early line defense against biological invasion, to lipopolysaccharides (LPS) results in significant cell population shifts in terms of decreased average velocities when compared to control cells. Confocal microscopy, atomic force microscopy (AFM) and Raman spectroscopy are utilized as complementary tools to study changes in the macrophage cells upon exposure to LPS and help explain the velocity changes observed within the microfluidic chip.
\end{abstract}

Key words: microfluidic, macrophage, label-free, optical pressure, lipopolysaccharide

\section{Introduction}

Optical chromatography $(\mathrm{OC})$ is a label-free sensing and separation technique which relies on the balance of optical and drag forces inside the channel of a microfluidic chip. [1] OC employs a mildly focused laser beam to exert optical pressure on particles carried by a fluid, enabling the sensing (static mode) and separation (flow-through mode) of live cells based on their size, shape, refractive index, surface morphology, and cellular structure. Major advantages associated with OC separation and analysis of live cells include label-free and rapid adaptation to novel or altered targets.

Innate immunity is the immediate response of the immune system to antigens invading the human body. Macrophages are a subset of innate immune cells which play a key role in the early line defense against pathogen invasion. We are investigating the feasibility of using $\mathrm{OC}$ to detect biological invasion inside the human body well before the appearance of symptoms, by measuring changes in optical pressure for macrophage cells after exposure to pathogens.

\section{Experimental}

At the heart of $O C$ is a 5-Layer microfluidic chip. This chip contains a 3D hydrodynamic focusing nozzle (Figure 1) that connects to three sheath flow channels, an injection channel at the center of the nozzle, and an exit channel which can be branched into two or more channels allowing cell sorting and collection. The flow is controlled pneumatically and the zig-zag configuration of the injection channel is essential to entraining injected cells at low flow rates.

The laser used in this study is a continuous wave (CW) $1064 \mathrm{~nm}$ ytterbium fiber laser. The macrophage cell line is ATCC TIB-186. A custom software written in Labview controls laser power and flowrates in all the channels, and allows the simultaneous measurements of cell average velocity, size, eccentricity, and orientation. Two modes of operation in OC are (1) static mode: balancing each cell at a predetermined position using a fixed laser power, and recording the flow rate which results in a net zero force exerted on each cell $\left(F_{O P}=\right.$ $\left.F_{d r a g}\right)$; (2) flow-through mode: fixing both laser power and flow rate while keeping $F_{O P}<F_{d r a g}$, and measuring the average velocities of cells flowing through a predetermined window. 


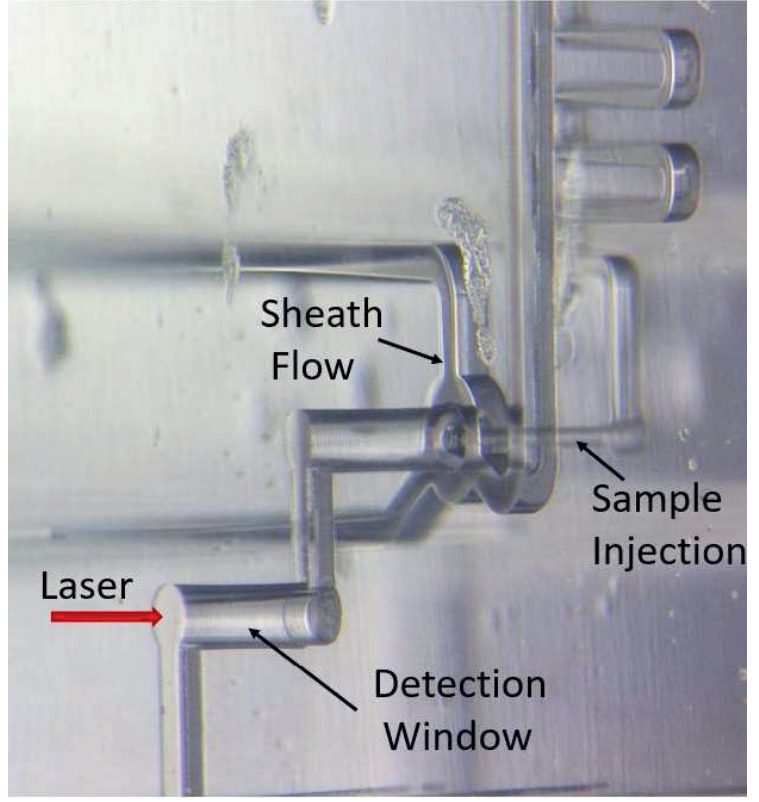

Fig. 1. $3 D$ hydrodynamic focusing nozzle and detection window.

\section{Results}

Upon exposure of macrophages to lipopolysaccharides (LPS), a major component of the outer membrane of gram-negative bacteria, OC in flow-through mode rapidly detects significant cell population shifts in terms of decreased average velocities when compared to control cells (Fig.2 (a)). The stimulated cells exhibit a slight increase in size as compared to the control cells (Fig.2 (b)). The results indicate, however, that the decrease in average velocities measured for stimulated cells, which reflect the higher optical pressures experienced by these cells, cannot be accounted for simply by the small increase in cell size alone.

Using picoNewton forces on a JPK Atomic Force Microscope (AFM), the images and force maps of live macrophages have been successfully acquired. Compared to the control cells, the stimulated cells display an overall increase in surface roughness and a decrease in the Young's modulus in areas surrounding the nucleus, suggesting the redistribution of $\mathrm{F}$ actin. Confocal microscopy images of control and stimulated macrophages stained with actintracker Alexa Fluor 488 Phalloidin and DAPI corroborate the findings from AFM; stimulated cells exhibit $\mathrm{F}$-actin redistribution. In some areas surrounding the nucleus, F-actin intensity is diminished, but in other areas the clustering of F-actin is observed. Finally, Raman spectra obtained on LPS stimulated cells exhibit greater signal enhancement corresponding to the reduced state for cytochrome, and the redistribution of lipid mapped by $\mathrm{C}-\mathrm{H}$ functionality.
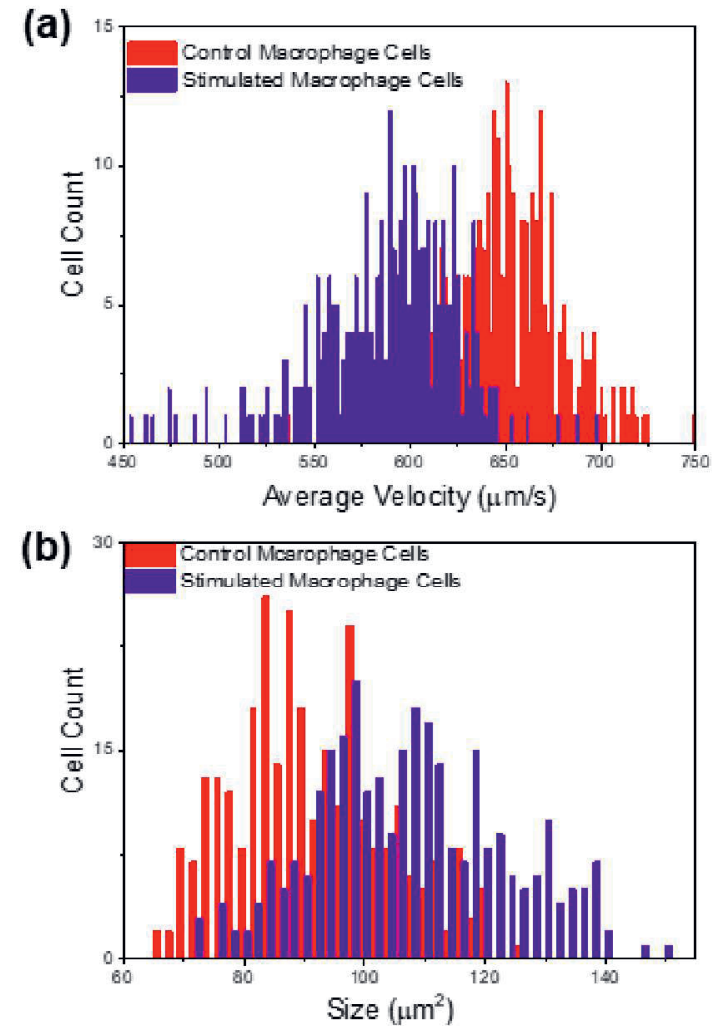

Fig. 2. Detecting response of macrophage cells to LPS using flow-through OC.

It is clear that other factors such as increased cell surface roughness and changes in cellular structure also contribute to the cell population shifts upon stimulation, as manifested by the observed decrease in average velocities detected by OC.

\section{Conclusions}

$\mathrm{OC}$ on a microfluidic platform is a novel labelfree approach to live cell sensing and analysis. OC rapidly and sensitively detects cell population shifts in flow-through mode upon exposure of macrophages to LPS. The cell population shift towards lower average velocities arises from changes in cell surface morphology and cellular structure, as supported by results derived from AFM, confocal microscopy, and Raman spectroscopy.

\section{References}

[1] C.G. Hebert, S. Hart, T.A. Leski, A. Terray, Q. Lu, TLabel-Free Detection of Bacillus anthracis Spore Uptake in Macrophage Cells Using Analytical Optical force Measurements, Analytical Chemistry 89, 10296-10302 (2017); doi: 10.1021/acs.analchem.7b01983 